\title{
Oxidative damage and mitochondrial injuries differ following pneumoperitoneum pressure in rabbit models of varying degrees of hydronephrosis
}

\author{
WEI LI ${ }^{1 *}$, SHENG ZHAO ${ }^{2 *}$, FAN CHENG ${ }^{2}$, TING RAO $^{2}$, WEIMIN YU ${ }^{2}$, \\ YUAN RUAN $^{2}$, RUN YUAN ${ }^{2}$ and XIAOBING YAO ${ }^{2}$ \\ Departments of ${ }^{1}$ Anesthesiology and ${ }^{2}$ Urology, Renmin Hospital of Wuhan University, Wuhan, Hubei 430000, P.R. China
}

Received October 31, 2017; Accepted January 26, 2018

DOI: $10.3892 / \mathrm{mmr} .2018 .8665$

\begin{abstract}
The influence of intraabdominal pressure which is necessary to maintain the operating area during the surgery cannot be ignored especially on the kidneys. Many articles have reported the effect of intraabdominal pressure on normal kidneys. However, the influence of intraabdominal pressure on hydronephrosis kidneys is rarely studied. The aim of the present study was to clarify whether intraabdominal pressure tolerance is modified in various degrees of kidney hydronephrosis by evaluating oxidative damage and mitochondrial injuries. A total of 72 rabbits were randomly divided into three groups (groups N, M and S, which represented rabbits with no, mild and severe hydronephrosis, respectively). Rabbits in groups $\mathrm{M}$ $(n=24)$ and $S(n=24)$ underwent a surgical procedure inducing mild or severe hydronephrosis, respectively. Subsequently, rabbits in all groups were allocated to 4 subgroups (N0-N3, M0-M3 and S0-S3) consisting of 6 rabbits each. Groups 0 to 3 were, respectively, subjected to intraabdominal pressures of $0,5,10$ and $15 \mathrm{mmHg}$. Oxidative damage was assessed by analyzing levels of reactive oxygen species (ROS), superoxide dismutase (SOD), malondialdehyde (MDA), glutathione peroxidase (GSH-Px), catalase (CAT) and lactate (LD). Mitochondrial injuries were assessed based on mitochondrial membrane potential (MMP) alterations, mitochondrial structure and cytochrome $c$ (cytc) protein expression, as measured by JC-1 staining, electron microscopy and western blotting, respectively. Oxidative damage and mitochondrial injuries were noticeably exacerbated in group $\mathrm{N}$ and $\mathrm{M}$ with increased
\end{abstract}

Correspondence to: Professor Fan Cheng, Department of Urology, Renmin Hospital of Wuhan University, 99 Zhang Zhidong Road, Wuhan, Hubei 430000, P.R. China

E-mail: urology1969@aliyun.com

*Contributed equally

Key words: oxidative damage, mitochondrial injury, hydronephrosis, pneumoperitoneum pressure, kidney levels of ROS, MDA and LD, decreased levels of SOD, GSH-Px, CAT and MMP, mitochondrial vacuolization and higher expression of cytc when the intraabdominal pressure reached $15 \mathrm{mmHg}$. In group $\mathrm{S}$, these alterations occurred at pressures of 10 and $15 \mathrm{mmHg}$. Therefore, it was concluded that in rabbits exposed to pneumoperitoneal pressure, kidneys with severe hydronephrosis were more likely to suffer from oxidative damage and mitochondrial injuries compared with kidneys with mild hydronephrosis and normal kidneys.

\section{Introduction}

With the rapid development of surgical technology, laparoscopic surgery has gradually replaced the traditional open operation owing to its improved cosmetic results, shorter post-operative hospital stays, reduced pain and faster return to preoperative condition (1-3). Despite these benefits, laparoscopic procedure can produce adverse effects secondary to intraabdominal pressure and an increasing number of studies have demonstrated that high intraabdominal pressure caused by carbon dioxide during laparoscopic surgery may have adverse effects on splanchnic organs $(4,5)$. Clinical and experimental studies have established that the increase in intraabdominal pressure that develops depending on the degree of pneumoperitoneum during laparoscopic surgery may cause hypoperfusion of intraabdominal organs $(6,7)$. Increases in ischemia and the oxidative stress response have been observed with pneumoperitoneum-dependent impairment of organ perfusion. Following desufflation, reperfusion injury occurred with a decrease in intraabdominal pressure $(8,9)$.

Kidneys, as important splanchnic organs, are inevitably affected by intraabdominal pressure. Some animal experiments have demonstrated that high and erratic elevations of intraabdominal pressure can decrease venous return, compress the renal vasculature and cause systemic hormonal changes, which eventually decrease renal blood flow, urinary output and glomerular filtration rate significantly (10). Other studies have observed increases in renal ischemia and oxidative stress response with increased intraabdominal pressure $(11,12)$. Although abdominal deflation at the end of laparoscopic procedures reduces intraabdominal pressure and increases renal perfusion, damage from the ischemic injury remains. 
However, the majority of studies concerning pneumoperitoneum pressure damage are based on normal kidneys, whereas certain patients who undergo laparoscopic surgery may also exhibit a certain degree of kidney obstruction $(13,14)$. The influence of intraabdominal pressure on hydronephrosis kidneys, caused by stones, tumors or congenital anomalies, cannot be ignored. A kidney with hydronephrosis exhibits a thinner renal cortex, its blood perfusion is already subnormal and hydronephrosis itself has adverse effects on renal tubule function. Therefore, it was hypothesized that hydronephrotic kidneys may have an increased susceptibility to injury as a result of increased kidney pressure during endourological procedures. The present study investigated whether tolerance to pneumoperitoneum pressure differs in rabbit models of no, mild and severe hydronephrosis by evaluating oxidative damage and mitochondrial injuries.

\section{Materials and methods}

Animals and groups. A total of 72 adolescent male New Zealand rabbits (6 months old, weighing 2.0-2.5 kg) were purchased from the Wuhan Institute of Biological Products Co., Ltd. (Wuhan, China). Rabbits were allowed to adapt to the laboratory environment for one week prior to the beginning of the experiment. The rabbits were housed in standard cages with free access to tap water and food, at a temperature of $18-25^{\circ} \mathrm{C}$ and relative humidity of $45-55 \%$. The entire procedure complied with the guidelines for the Care and Use of Laboratory Animals (15) and the Ethical and Research Committee of Wuhan University Medical School (Wuhan, China) approved the animal study.

The rabbits were randomly divided into three groups consisting of 24 rabbits each: Normal (N), mild (M) and severe (S) hydronephrosis groups. For the $\mathrm{M}$ and $\mathrm{S}$ groups, rabbits underwent surgical procedures to induce mild or severe hydronephrosis. For the $\mathrm{N}$ group, rabbits received a sham surgical procedure and no hydronephrosis was induced. Following surgery, the rabbits were randomly assigned to 4 subgroups (N0-N3, M0-M3 and S0-S3) consisting of 6 rabbits each. Rabbits in groups 0-3 were insufflated with carbon dioxide in their abdomens to maintain intraabdominal pressures of 0,5 , 10 and $15 \mathrm{mmHg}$, respectively.

Surgical manipulation. The surgical model by Wen et al (16) was employed. Briefly, the rabbits were anesthetized with $40 \mathrm{mg} / \mathrm{kg}$ intraperitoneal sodium pentobarbital at room temperature. The left ureter, left lumbar vein and psoas muscle were exposed through a midline abdominal incision. Separately, for the mild and severe hydronephrosis groups, the proximal ureter was buried in a 2 - and $4-\mathrm{cm}$ notch within the psoas muscle. For the normal group, only a midline abdominal incision was performed, and the abdomen was then closed (sham procedure). After 2 weeks, B-ultrasonography was used to confirm hydronephrosis. In the $\mathrm{M}$ and $\mathrm{S}$ groups, respectively, pyelic distention levels of $0.95 \pm 0.27$ and $1.69 \pm 0.34 \mathrm{~cm}$, and parenchymal thicknesses of $0.33 \pm 0.09$ and $0.22 \pm 0.05 \mathrm{~cm}$, were observed. A second laparotomy was then performed where, following the anesthetization, a 0.5 -cm-long incision was made in the left abdomen. A 10-gauge Veress needle was inserted into the peritoneal cavity through the incision and the other side of the Veress needle was connected to a $\mathrm{CO}_{2}$ insufflator (Stryker Endoscopy, Kalamazoo, MI, USA). Subsequently, the incision was sutured to prevent $\mathrm{CO}_{2}$ leakage from the abdomen. The pressure for the 0-3 subgroups was set at 0,5, 10 and $15 \mathrm{mmHg}$, respectively, for the $\mathrm{N}, \mathrm{M}$ and $\mathrm{S}$ groups. After $1 \mathrm{~h}$ of insufflation, the pneumoperitoneum was released, the psoas muscle obstruction was relieved and the abdomen was sutured closed. Rabbits were sacrificed using $150 \mathrm{mg} / \mathrm{kg}$ pentobarbital (20\%) through the ear marginal vein injection after $24 \mathrm{~h}$, and the left kidneys were collected for biochemical and histological evaluations.

Determination of reactive oxygen species (ROS). Kidney tissue samples were initially homogenized using a T25 digital Ultra-Turrax ${ }^{\circledR}$ disperser (IKAH-Labortechnik, Staufen, Germany) in $100 \mathrm{mmol} / \mathrm{l} \mathrm{PBS}$ and centrifuged at $13,000 \mathrm{x} \mathrm{g}$ and $4^{\circ} \mathrm{C}$ for $10 \mathrm{~min}$ (Heraeus Biofuge Primo $\mathrm{R}$ centrifuge), after which the supernatants were collected for detection. The homogenized supernatants were incubated with 4-amino-5-methylamino-2',7'-difluorofluorescein (1 mmol/l; Nanjing Jiancheng Bioengineering Institute, Nanjing, China) for $30 \mathrm{~min}$ at $37^{\circ} \mathrm{C}$. The absorbance was detected at $500 \mathrm{~nm}$ using an automatic microplate reader (Multiskan MK3; Thermo Fisher Scientific, Inc., Waltham, MA, USA). The results are expressed as fluorescence intensity/mg protein (A.U./mg prot).

Detection of superoxide dismutase (SOD). Tissues were homogenized using a T25 digital Ultra-Turrax disperser in Tris buffer ( $\mathrm{pH}$ 7.4) containing butylated hydroxytoluene to prevent new lipid peroxidation that may occur during homogenization. Samples were centrifuged at $13,000 \times \mathrm{g}$ and $4^{\circ} \mathrm{C}$ for $20 \mathrm{~min}$ (Heraeus Biofuge Primo R centrifuge), after which the supernatants were collected. Total Superoxide Dismutase (T-SOD) assay kit (A001-1-1; Nanjing Jiancheng Bioengineering Institute) was used for determining SOD levels. The xanthine oxidase method (17) was used for detection and the absorbance was detected at a wavelength of $550 \mathrm{~nm}$ using an automatic microplate reader (Multiskan MK3). The results are expressed as units/mg protein (U/mg prot).

Measurement of malondialdehyde (MDA). First, kidney tissue samples were homogenized using a T25 digital Ultra-Turrax ${ }^{\circledR}$ disperser (IKAH-Labortechnik) in normal saline and centrifuged at $13,000 \mathrm{x} \mathrm{g}$ and $4^{\circ} \mathrm{C}$ for $10 \mathrm{~min}$ (Heraeus Biofuge Primo R centrifuge), then the concentration of malondialdehyde (MDA) was measured using an assay kit (A003-1; Nanjing Jiancheng Bioengineering Institute). Briefly, MDA reacts with thiobarbituric acid to form a stable chromophoric product, which was subsequently detected with an automatic microplate reader (Multiskan MK3) at a wavelength of $532 \mathrm{~nm}$. The difference in absorption reflects different MDA concentration in each sample. Results are expressed as units/ml (U/ml).

Detection of catalase (CAT) activity. Tissues were homogenized and centrifuged at $13,000 \mathrm{x} \mathrm{g}$ and $4^{\circ} \mathrm{C}$ for $10 \mathrm{~min}$. Catalase (CAT) assay kit (A007-1; Nanjing Jiancheng Bioengineering Institute) was used for determining CAT levels. The CAT levels of the homogenates were assayed at 520 
and $535 \mathrm{~nm}$ using an automatic microplate reader (Multiskan MK3). The results were expressed as $\mathrm{U} / \mathrm{ml}$.

Glutathione peroxidase (GSH-Px) assay. Tissues were homogenized and centrifuged at $13,000 \mathrm{xg}$ and $4^{\circ} \mathrm{C}$ for $10 \mathrm{~min}$. A Glutathione Peroxidase assay kit (A006; Nanjing Jiancheng Bioengineering Institute) was used. According to the manufacturer's protocol, GSH reacts with 5,5'-dithiobis-2-nitrobenzoic acid and the absorbance spectrum of the product has a maximum absorbance at a wavelength of $410 \mathrm{~nm}$. The results were expressed as units/g protein (U/g prot).

Lactate ( $L D)$ levels. Lactate (LD) is the product of anaerobic respiration and LD levels indicate the extent of hypoxia. Homogenates were prepared after homogenation and centrifugation at $10,000 \mathrm{x}$ g and $4^{\circ} \mathrm{C}$ for $10 \mathrm{~min}$. Then a lactate assay kit (A018; Nanjing Jiancheng Bioengineering Institute) was used for LD detection. The results were expressed as nanomoles/g protein (nmol/g prot).

Mitochondrial membrane potential (MMP) detection. JC-1, a cationic dye, is used as an indicator of mitochondrial potential. It represents mitochondrial potential-dependent accumulation, which is detected based on a fluorescence emission shift from green to red. Briefly, fresh renal tissue was cleaned with $0.9 \%$ normal saline and was subsequently digested in trypsin solution (Beyotime Institute of Biotechnology, Haimen, China) at $37^{\circ} \mathrm{C}$ for $\sim 20 \mathrm{~min}$. The digestion was terminated by the addition of $30 \%$ bovine serum (Hangzhou Sijiqing Biological Engineering Materials Company, Hangzhou, China). Suspension cells were centrifuged at $2,000 \times \mathrm{g}$ and $4^{\circ} \mathrm{C}$ for $4 \mathrm{~min}$ and washed with PBS three times. For JC-1 staining, the cells $\left(\sim 3 \times 10^{5} / \mathrm{ml}\right)$ were loaded with 1X JC-1 (Beyotime Institute of Biotechnology) at $37^{\circ} \mathrm{C}$ for $20 \mathrm{~min}$ and then washed and analyzed via flow cytometry (FACSCalibur; BD Biosciences, Franklin Lakes, NJ, USA) and related software (Flowjo version 7.6.1; FlowJo LLC, Ashland, OR, USA).

Mitochondrial structure by electron microscopy. Minute pieces of renal cortex were sectioned and fixed in $2.5 \%$ glutaraldehyde at $4^{\circ} \mathrm{C}$ overnight, washed with $0.1 \mathrm{M}$ PBS ( $\mathrm{pH} 7.2$ ) and subsequently fixed in $2 \%$ osmium tetroxide at $4^{\circ} \mathrm{C}$ for $2 \mathrm{~h}$. The tissues were dehydrated in graded alcohol and then embedded in epoxy resin at $45^{\circ} \mathrm{C}$ for $12 \mathrm{~h}$. All tissues samples were sectioned at $50 \mathrm{~nm}$ and washed again with distilled water and prior to being stained with uranyl acetate $(2 \%)$ and lead citrate $(10 \%)$ for $30 \mathrm{~min}$ at $25^{\circ} \mathrm{C}$, respectively. Then samples were visualized under a transmission electron microscope (H-600; Hitachi, Ltd., Tokyo, Japan). The mitochondrial ultramicrostructure changes were observed in five random fields of view for each section.

Western blotting. Cytochrome $c$ (cytc) expression levels in left rabbit kidney tissues were detected using western blotting. Briefly, tissues were homogenized with a T25 digital Ultra-Turrax disperser in radioimmunoprecipitation assay buffer (Beyotime Institute of Biotechnology) containing phenylmethylsulfonyl fluoride (Beyotime Institute of Biotechnology) and were centrifuged at $14,000 \times \mathrm{g}$ and $4^{\circ} \mathrm{C}$ for $20 \mathrm{~min}$. The bicinchoninic acid method was used to detect the protein concentration. Then $\sim 50 \mu \mathrm{g}$ proteins in each group were added onto the gels per lane for detection, then samples were subjected to $12 \%$ SDS-PAGE and were transferred onto polyvinylidene difluoride membranes for $1 \mathrm{~h}$ at $200 \mathrm{~mA}$. The membranes were blocked with $5 \%$ dried skimmed milk at room temperature $\left(\sim 25^{\circ} \mathrm{C}\right)$ for $1 \mathrm{~h}$ and were subsequently incubated with mouse primary antibodies against cytc (NB100-56503; 1:5,000; Novus Biologicals, LLC, Littleton, CO, USA) and $\beta$-actin (ab28052, 1:5,000; Abcam, Cambridge, UK) overnight at $4^{\circ} \mathrm{C}$. After washing, a goat anti-mouse secondary antibody (P/N 925-32210; 1:10,000; LI-COR Biosciences, Lincoln, NE, USA) conjugated to IRDye $800 \mathrm{CW}$ was added and incubated for $1 \mathrm{~h}$ at room temperature. The signal was quantified using a western blot detection system (Odyssey Infrared Imaging; LI-COR Biosciences). Semi-quantitative analysis was conducted (Image Studio version 5.2.5; LI-COR Biosciences) for the corresponding protein expression levels.

Statistical analysis. Data are presented as the mean + standard deviation. All analyses were performed in duplicate. The statistical software package SPSS version 19 (IBM Corp., Armonk, NY, USA) was used for statistical analysis. One-way analysis of variance and Tukey's post hoc test were used for statistical comparisons. $\mathrm{P}<0.05$ was considered to indicate a statistically significant difference.

\section{Results}

Levels of ROS, SOD, MDA, GSH-Px, CAT and LD in hydronephrotic kidney tissues following pneumoperitoneum. In group N, the ROS, SOD, MDA, GSH-PX, CAT and LD levels were comparable when subjected to intraabdominal pressures of 0,5 and $10 \mathrm{mmHg}$ ( N0, N1 and $\mathrm{N} 2$ groups, respectively; P>0.05; Fig. 1A). However, when the pressure reached $15 \mathrm{mmHg}$ (N3 group), the ROS, MDA and LD levels significantly increased, and the SOD GSH-Px and CAT levels decreased, compared with the N0 group ( $\mathrm{P}<0.05$; Fig. 1A). In group $\mathrm{M}$, similar results were observed (Fig. 1B). In group $\mathrm{S}$, the ROS, SOD, MDA, GSH-Px, CAT and LD levels were comparable at pressures of 0 and $5 \mathrm{mmHg}$ ( $\mathrm{S} 0$ and $\mathrm{S} 1$ groups, respectively); however, at 10 and $15 \mathrm{mmHg}$ (S2 and $\mathrm{S} 3$ groups, respectively), the ROS, MDA and LD levels significantly increased and the SOD, GSH-Px and CAT levels significantly decreased compared with the S0 group $(\mathrm{P}<0.05$; Fig. $1 \mathrm{C})$. Furthermore, marginal increases in the ROS, MDA and LD levels and decreases in the SOD, GSH-Px and CAT levels were observed with the increasing degree of hydronephrosis in groups that suffered no intraabdominal pressure (N0, M0 and S0 groups; Fig. 1).

Alterations in MMP levels of hydronephrotic kidneys following pneumoperitoneum. The present study measured MMP to determine mitochondrial injuries with JC-1. When MMP levels are high, JC-1 primarily exists in the mitochondrial matrix as a polymer, which emits red fluorescence (excitation wavelength of $525 \mathrm{~nm}$ and emission wavelength of $590 \mathrm{~nm}$ ). When the MMP levels are low, JC-1 primarily exists in the cytoplasm as monomers, which emits green fluorescence (excitation wavelength of $490 \mathrm{~nm}$ and emission wavelength of $540 \mathrm{~nm}$ ). Thus, alterations in the ratio of red to green fluorescence intensity 




B



C

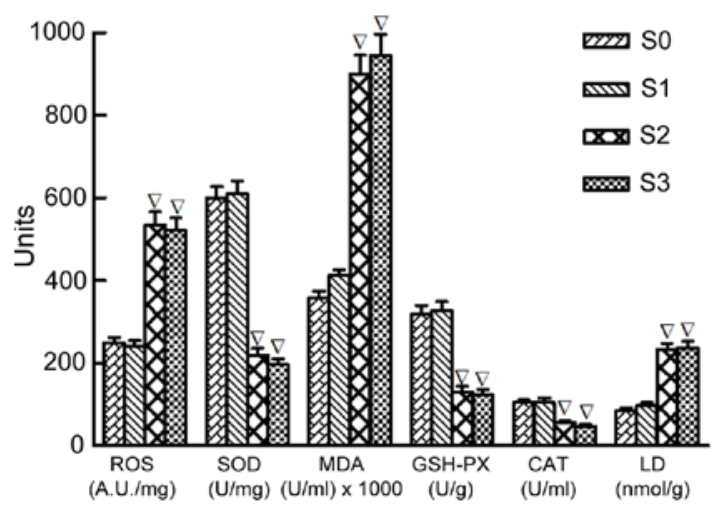

Figure 1. Levels of ROS, SOD, MDA, GSH-Px, CAT and LD in normal kidneys and kidneys with mild and severe hydronephrosis under different intraabdominal pressures. (A) Normal rabbit kidneys were represented by group N. N0, N1, N2 and N3 represented normal kidneys subjected to intraabdominal pressures of $0,5,10$ and $15 \mathrm{mmHg}$, respectively. (B) Rabbi kidneys with mild hydronephrosis were represented by group M. M0, M1, M2 and M3 represented rabbits with mild hydronephrosis subjected to intraabdominal pressures of $0,5,10$ and $15 \mathrm{mmHg}$, respectively. (C) Rabbit kidneys with severe hydronephrosis were represented by group S. S0, S1, S2 and S3 represented rabbits with severe hydronephrosis subjected to intraabdominal pressures of $0,5,10$ and $15 \mathrm{mmHg}$, respectively. ${ }^{\#} \mathrm{P}<0.05$ vs. N0 group; ${ }^{*} \mathrm{P}<0.05$ vs. M0 group; ${ }^{\nabla} \mathrm{P}<0.05$ vs. S0 group. ROS, reactive oxygen species; SOD, superoxide dismutase; MDA, malondialdehyde; GSH-Px, glutathione peroxidase; CAT, catalase; LD, lactate.

represents the alterations in MMP levels; a decrease in MMP levels is considered to be an early apoptotic event (18).

MMP values were expressed as the ratio of red fluorescence intensity to the green fluorescence intensity, indicated in Q2 and Q4, respectively. In groups $\mathrm{N}$ and M, the MMP levels were similar in both groups at 0,5 and $10 \mathrm{mmHg}$, but decreased when the pressure reached $15 \mathrm{mmHg}$. In group $\mathrm{S}$, the MMP levels were similar when the pressure was 0 and $5 \mathrm{mmHg}$, but significantly decreased when the pressure was 10 and $15 \mathrm{mmHg}$. Furthermore, no significant differences were observed between the S2 and S3 groups (Fig. 2). Furthermore, a marginal decrease in MMP levels was observed with the increased extent of hydronephrosis in groups that suffered no intraabdominal pressure (N0, M0 and S0 groups; Fig. 2).

Mitochondrial ultramicrostructure changes. Transmission electron microscopy was used to detect the mitochondrial ultramicrostructure in renal cells. The present study investigated the mitochondrial damage by counting the percentages of swollen and vacuolar mitochondria in the different groups. In group $\mathrm{N}$, no swollen and vacuolar mitochondria were observed at pressures of 0,5 and $10 \mathrm{mmHg}$. However, when the pressure increased to $15 \mathrm{mmHg}$, the percentage of swollen and vacuolar mitochondria increased. In group $\mathbf{M}$, the percentage of swollen and vacuolar mitochondria were comparable at 0,5 and $10 \mathrm{mmHg}$, but increased significantly when the pressure was $15 \mathrm{mmHg}$. In group $\mathrm{S}$, the percentage of swollen and vacuolar mitochondria were similar at 0 and $5 \mathrm{mmHg}$, but significantly increased at pressures of 10 and $15 \mathrm{mmHg}$. No significant differences were observed between S2 and S3 groups (Fig. 3). Furthermore, the percentage of swollen and vacuolar mitochondria increased relatively with the increase in the extent of hydronephrosis in rabbits suffering no intraabdominal pressure (N0, M0 and S0 groups; Fig. 3).

Expression of cytc. Western blot analysis demonstrated that cytc protein expression in group $\mathrm{N}$ was comparable when subjected to intraabdominal pressures of 0,5 and $10 \mathrm{mmHg}$, but significantly increased when the pressure was $15 \mathrm{mmHg}$. A similar result was observed in group $M$. In group $S$, cytc protein expression was comparable at intraabdominal pressures of 0 and $5 \mathrm{mmHg}$, and significantly increased at pressures of 10 and $15 \mathrm{mmHg}$ (Fig. 4).

\section{Discussion}

The present study demonstrated that oxidative damage and mitochondrial injuries occurred in obstructed kidneys during pneumoperitoneum. It was also demonstrated that oxidative damage and mitochondrial injuries were more severe with a greater extent of obstruction, meaning that severely obstructed kidneys may exhibit reduced cell tolerance to intraabdominal pressure and that they are more likely to suffer oxidative damage and mitochondrial injuries.

Pneumoperitoneum, although generally considered to be essential for adequate exposure in laparoscopic surgery, has been reported to exert adverse effects on renal physiology, particularly under high intraabdominal pressure $(>10 \mathrm{mmHg})(19,20)$. Numerous factors contribute to these adverse effects. Wiesenthal et al (21) emphasized that high intraabdominal pressure may noticeably decrease renal blood flow. Borba et al (22) demonstrated that the renin-angiotensin-aldosterone system also affects renal blood flow. These effects may eventually cause hypoxic-ischemic damage, and when intraabdominal pressure is removed, 

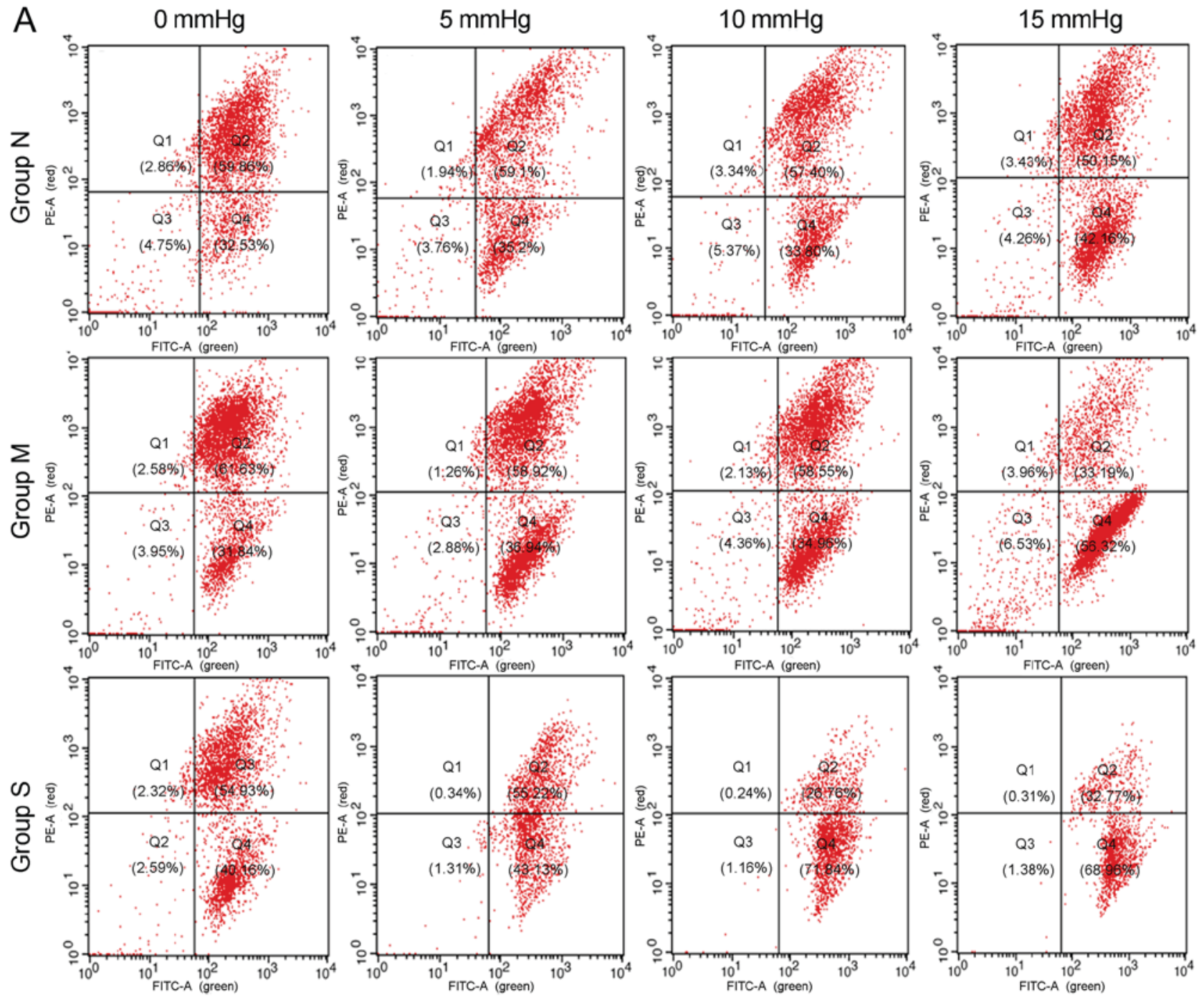

B



Figure 2. MMP of renal cells in normal kidneys and kidneys with mild and severe hydronephrosis under different intraabdominal pressures. (A) MMP analysis by flow cytometry. PE-A represented red fluorescence and FITC-A represented green fluorescence. MMP values were expressed as the ratio of red fluorescence intensity to the green fluorescence intensity, indicated in Q2 and Q4, respectively. (B) Ratios of red fluorescence intensity to the green fluorescence intensity in rabbits with no, mild and severe hydronephrosis under different intraabdominal pressures. Normal rabbit kidneys were represented by group N. N0, N1, N2 and $\mathrm{N} 3$ represented normal kidneys subjected to intraabdominal pressures of $0,5,10$ and $15 \mathrm{mmHg}$, respectively. Rabbit kidneys with mild hydronephrosis were represented by group M. M0, M1, M2 and M3 represented rabbits with mild hydronephrosis subjected to intraabdominal pressures of $0,5,10 \mathrm{and} 15 \mathrm{mmHg}$, respectively. Rabbit kidneys with severe hydronephrosis were represented by group S. S0, S1, S2 and S3 represented rabbits with severe hydronephrosis subjected to intraabdominal pressures of $0,5,10$ and $15 \mathrm{mmHg}$, respectively. ${ }^{*} \mathrm{P}<0.05$ vs. N0 group; ${ }^{*} \mathrm{P}<0.05$ vs. M0 group; ${ }^{7} \mathrm{P}<0.05$ vs. S0 group. MMP, mitochondrial membrane potential; PE, phycoerythrin; FITC, fluorescein isothiocyanate; Q, quadrant. 

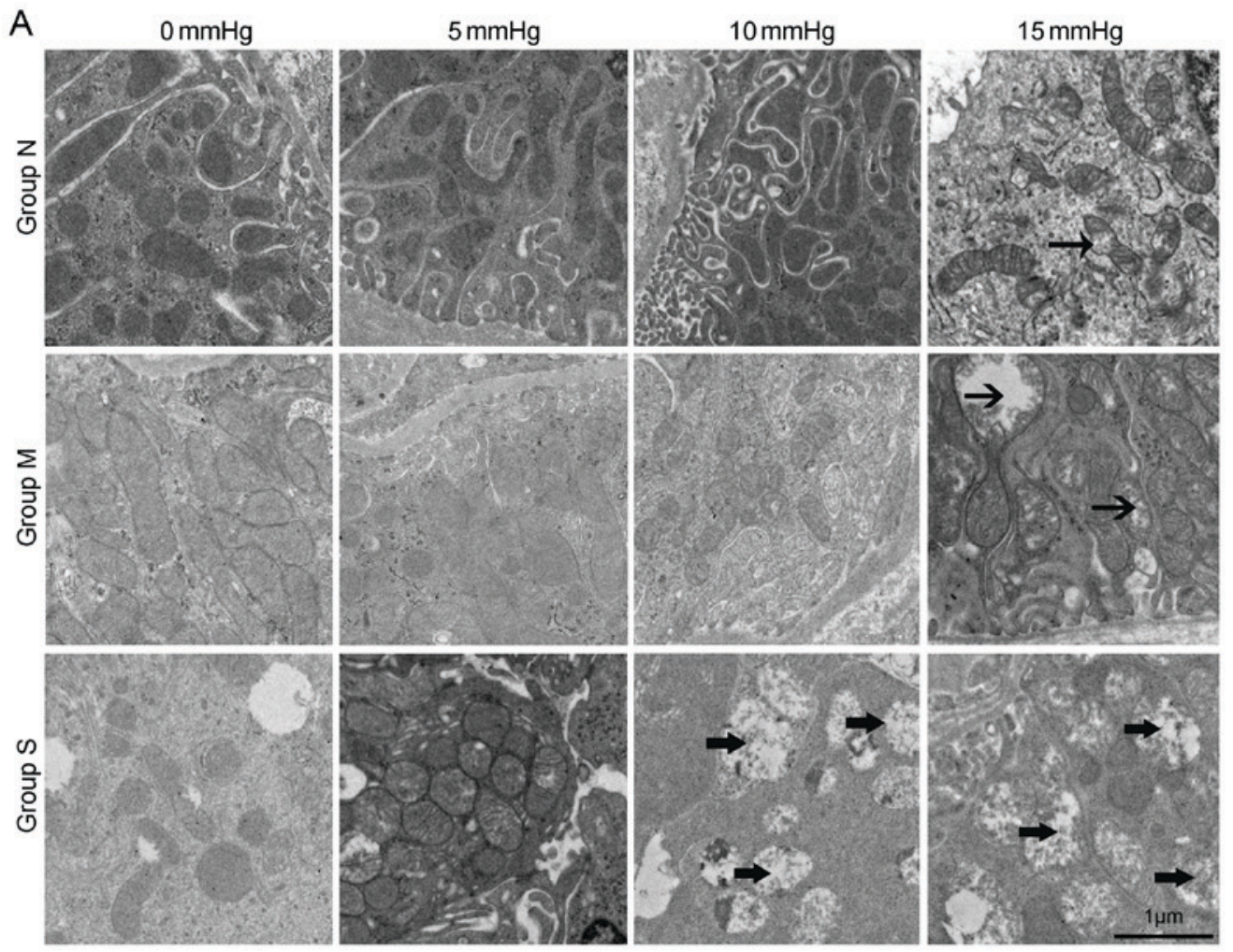

B

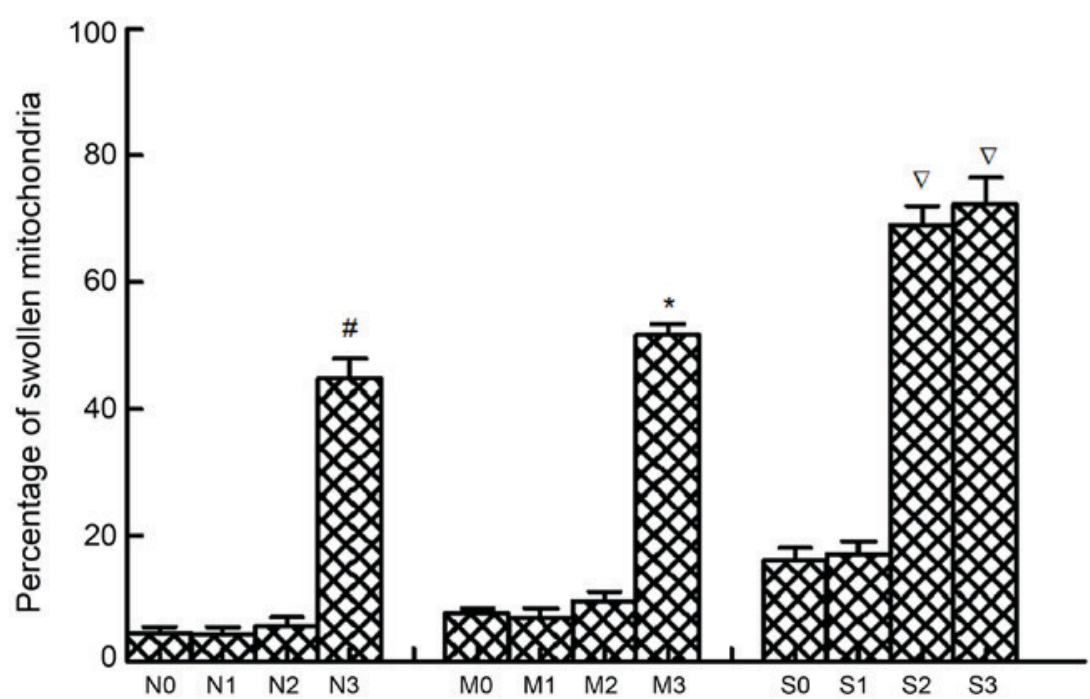

Figure 3. Ultrastructural alterations of mitochondria. (A) Swollen and vacuolar mitochondria in rabbit kidneys with no, mild and severe hydronephrosis, represented by groups $\mathrm{N}, \mathrm{M}$ and $\mathrm{S}$, respectively, were subjected to different intraabdominal pressures and examined by transmission electron microscopy. The arrows in this figure showed examples of swollen and vacuolar mitochondria in different groups. Magnification, x5,000. (B) Percentages of swollen and vacuolar mitochondria in each group. The 0-3 subgroups for groups $\mathrm{N}, \mathrm{M}$ and $\mathrm{S}$ represented kidneys subjected to intraabdominal pressures of $0,5,10$ and $15 \mathrm{mmHg}$, respectively. ${ }^{\#} \mathrm{P}<0.05$ vs. N0 group; ${ }^{\mathrm{P}}<0.05$ vs. $\mathrm{M} 0$ group; ${ }^{\nabla} \mathrm{P}<0.05$ vs. S0 group.

ischemia/reperfusion injury may occur. This type of injury has been associated with oxidative damage and mitochondrial injuries (23), and apoptosis or death eventually occurs $(24,25)$. As mentioned previously, certain patients undergoing surgery also present with a certain degree of kidney hydronephrosis. It has been reported that kidneys with hydronephrosis are more likely to suffer hypoxia problems $(26,27)$. According to our previous study, rabbit kidneys with severe hydronephrosis suffered acute kidney injury more readily compared with those with mildly nephrotic kidneys when exposed to pneumoperitoneal pressure (28). Another study demonstrated that severe hydronephrosis ( $\geq$ grade 3 ) led to prolonged pneumoperitoneum time and total operation time in laparoscopic radical nephroureterectomy (29). The prolonged operation time may lead to increased oxidative stress. Therefore, the present study investigated the effect of intraabdominal pressure based on kidneys with hydronephrosis.

The generation of ROS appears to be an important factor in tissue injury. To an extent, ROS content represents the degree of oxidative damage. Reperfusion reintroduces oxygen to the previously ischemic tissue, which results in a sudden burst of ROS (30). The primary mechanisms of ROS generation include anaerobic 
A

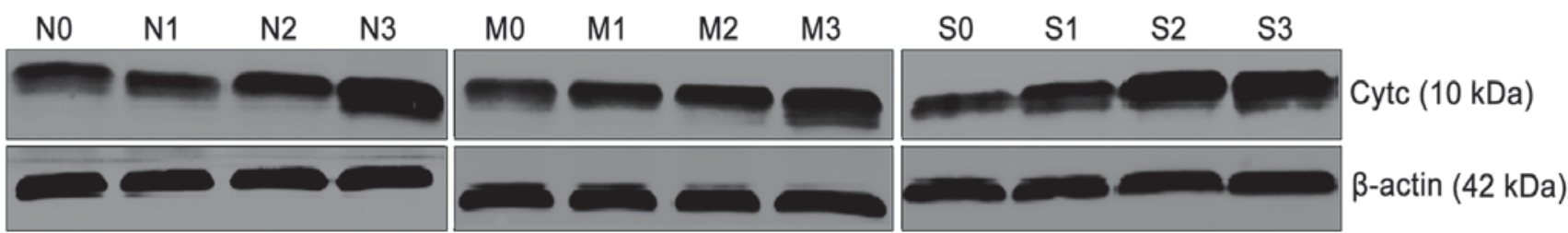

B

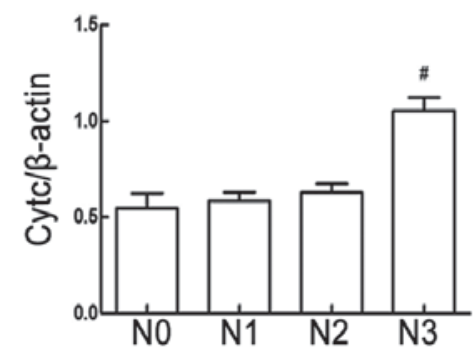

C

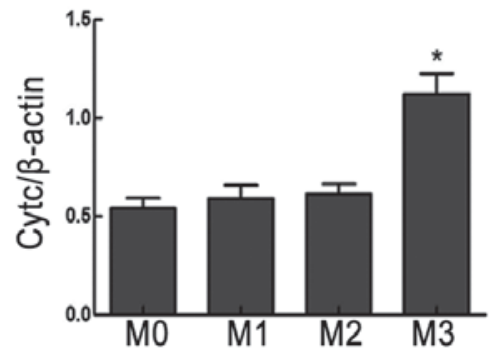

D

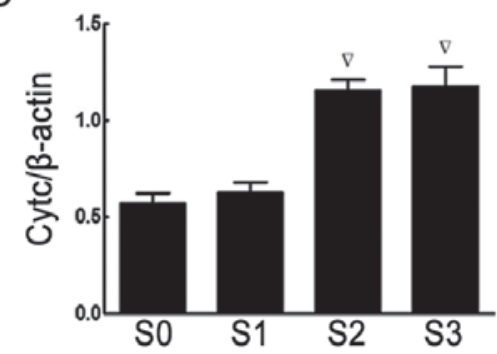

Figure 4. Cytc protein expression in normal kidneys and kidneys with mild and severe hydronephrosis under different intraabdominal pressures. (A) Representative western blots for the protein expression of cytc and $\beta$-actin in rabbit kidneys from groups N, M and S. (B) Relative expression of cytc in normal kidneys, represented by group N. (C) Relative expression of cytc in kidneys with mild hydronephrosis, represented by group M. (D) Relative expression of cytc in kidneys with severe hydronephrosis, represented by group $\mathrm{S}$. The 0-3 subgroups for groups $\mathrm{N}, \mathrm{M}$ and $\mathrm{S}$ represented kidneys subjected to intraabdominal pressures of $0,5,10$ and $15 \mathrm{mmHg}$, respectively. ${ }^{\sharp} \mathrm{P}<0.05$ vs. N0 group; ${ }^{*} \mathrm{P}<0.05$ vs. M0 group; ${ }^{\nabla} \mathrm{P}<0.05$ vs. S0 group. Cytc, cytochrome $c$.

mitochondrial respiration, activated neutrophils, increased xanthine oxidase levels and other factors (31). Mitochondrial membrane lipid peroxidation is considered to be an effect of ROS, and mitochondrial damage promotes the release of large amounts of ROS, which leads to a vicious cycle (32). MDA is a mediator of mitochondrial damage and leads to mitochondrial dysfunction through the inhibition of respiration and the inactivation of important mitochondrial enzymes (33). Therefore, by measuring MDA levels, the damage that ROS incurs on kidney tissue may be determined. SOD, CAT and GSH-Px enzymes constitute the primary components of the enzymatic antioxidant defense system against oxidative stress. SOD performs dismutation that leads to the formation of $\mathrm{H}_{2} \mathrm{O}_{2}$, which is subsequently removed by GSH-Px and CAT. GSH has also been reported to serve an additional role against lipid peroxidation (34). LD is a product of anaerobic respiration. When severe hypoxia occurs, tissues are not able to generate sufficient ATP from aerobic respiration and pyruvic acid is converted into LD by lactate dehydrogenase (35). In the present study, compared with the respective $0 \mathrm{mmHg}$ intraabdominal pressure groups, increased LD, ROS and MDA levels, and decreased SOD, GSH-Px and CAT levels, were observed in normal kidneys and kidneys with mild hydronephrosis subjected to $15 \mathrm{mmHg}$ intraabdominal pressure, and in rabbits with severe hydronephrosis subjected to 10 and $15 \mathrm{mmHg}$ pressure. The results indicated that kidneys with severe hydronephrosis suffered from oxidative damage more readily compared with normal kidneys and kidneys with mild hydronephrosis after being subjected to intraabdominal pressure.

A loss of MMP is reported to have an adverse effect on mitochondrial function (36). It is generally accepted that decreased MMP affects the opening of the mitochondrial permeability transition pore, which controls the release of apoptosis-activating factors, such as cytc. The release of cytc eventually leads to mitochondrial-dependent cell death (37). The current study observed a loss of MMP and higher cytc expression in normal kidneys and kidneys with mild hydronephrosis subjected to $15 \mathrm{mmHg}$ intraabdominal pressure, and in rabbits with severe hydronephrosis subjected to 10 and $15 \mathrm{mmHg}$ pressure, compared with the respective $0 \mathrm{mmHg}$ intraabdominal pressure groups, which indicated an increased severity of mitochondrial damage. This observation may be substantiated by the detection of ultrastructural alterations in the mitochondria. Chronic hypoxia has been reported to augment the quantity and the superficial area of mitochondria, which is conductive to oxygen diffusion. However, severe hypoxia may lead to mitochondrial deformation and swelling, and potentially the rupture of the outer membrane or spillover of the mitochondrial matrix (38). In the present study, the percentage of swollen and vacuolar mitochondria increased in the mild hydronephrosis and normal groups upon exposure to a pressure of $15 \mathrm{mmHg}$, and increased in the severe hydronephrosis group at pressures of 10 and $15 \mathrm{mmHg}$, compared with the respective $0 \mathrm{mmHg}$ groups.

In conclusion, the present study indicated that kidneys with severe hydronephrosis may be more likely to suffer mitochondrial injury than normal kidneys and kidneys with mild hydronephrosis following subjection to intraabdominal pressure. Additionally, marginal effects were identified with the increasing extent of hydronephrosis, even without increased intraabdominal pressure. This phenomenon may be explained as the effect hydronephrosis itself has on the kidneys rather than pneumoperitoneum.

However, if inherent limitations associated with animal models do apply, it would be irrelevant to consider whether similar intraabdominal pressures may be applied in humans as the level of cell tolerance to intraabdominal pressure may differ between humans and rabbits (39). The difference in the size of kidney samples between two species causes the pressure to be different per unit of kidney surface area. The current study indicated that oxidative damage and mitochondrial injuries are more likely to occur in obstructed kidneys 
during pneumoperitoneum and that the pressure should be kept lower when performing surgery. Based on the results of this study, further studies regarding the exact underlying mechanisms responsible for the decrease in tolerance to intraabdominal pressure are required. Furthermore, the insufflation of $\mathrm{CO}_{2}$ in experiments in the present study was performed at room temperature $\left(20-25^{\circ} \mathrm{C}\right)$ and dry $(0-5 \%$ relative humidity) conditions, and the intraabdominal pressures lasted for $1 \mathrm{~h}$. A retrospective analysis has provided evidence for the benefits of using humidified, warm $\mathrm{CO}_{2}$ vs dry, cool $\mathrm{CO}_{2}$ in surgery (40). However, Sammour et al (41) revealed that warming and humidification of insufflation gas had no effect on measures of oxidative stress compared with unwarmed and non-humidified controls. In another animal experiment, Akbulut et al (42) demonstrated that oxidative stress on kidneys increased with the prolonged duration of pneumoperitoneal pressure from 120-240 min, and the findings indicated that operating time should be limited to $<120$ min during laparoscopic surgery. The present study only determined the effect that different intraabdominal pressures had on obstructed kidneys. However, the duration of intraabdominal pressures and the properties of gas should also be investigated as other important factors.

In conclusion, the results of the current study demonstrated that rabbit kidneys with severe hydronephrosis were more likely to suffer oxidative damage and mitochondrial injuries than mild hydronephrosis and normal kidneys when they were exposed to pneumoperitoneal pressure. Therefore, intraabdominal pressure should be appropriately controlled and reduced during laparoscopic surgery in the context of kidney obstruction.

\section{Acknowledgements}

Not applicable.

\section{Funding}

The present study was supported by grants from the Nation Natural Science Fund Project of China (grant no. 81400698).

\section{Availability of data and materials}

The datasets used and/or analyzed during the current study are available from the corresponding author on reasonable request.

\section{Authors' contributions}

WL and SZ performed the experiment and were major contributors in writing the manuscript. FC conceived and designed the experiments. TR, WY and YR collected and analyzed the data. XY and RY supplied materials and analyzed the data. All authors read and approved the final manuscript.

\section{Ethics approval and consent to participate}

The present study was approved by the Ethical and Research Committee of Wuhan University Medical School (Wuhan, China).

\section{Consent for publication}

Not applicable.

\section{Competing interests}

The authors declare that they have no competing interests.

\section{References}

1. van der Toorn F, van den Hoek J, Wolffenbuttel KP and Scheepe JR: Laparoscopic transperitoneal pyeloplasty in children from age of 3 years: Our clinical outcomes compared with open surgery. J Pediatr Urol 9: 161-168, 2013

2. Seims AD, VanHouwelingen L, Mead J, Mao S, Loh A, Sandoval JA, Davidoff AM, Wu J, Wang WC and FernandezPineda I: Operative and immediate postoperative differences between traditional multiport and reduced port laparoscopic total splenectomy in pediatric patients. J Laparoendosc Adv Surg Tech A 27: 206-210, 2017.

3. Bartın MK, Kemik Ö, Çaparlar MA, Bostancı MT and Öner MÖ: Evaluation of the open and laparoscopic appendectomy operations with respect to their effect on serum IL-6 levels. Ulus Travma Acil Cerrahi Derg 22: 466-470, 2016.

4. Wever KE, Bruintjes MH, Warlé MC and Hooijmans CR: Renal perfusion and function during pneumoperitoneum: A systematic review and meta-analysis of animal studies. PLoS One 11: e0163419, 2016.

5. Sodha S, Nazarian S, Adshead JM, Vasdev N and Mohan-S G: Effect of pneumoperitoneum on renal function and physiology in patients undergoing robotic renal surgery. Curr Urol 9: 1-4, 2016.

6. de Geus HR, Betjes MG, Schaick Rv and Groeneveld JA: Plasma NGAL similarly predicts acute kidney injury in sepsis and nonsepsis. Biomark Med 7: 415-421, 2013.

7. Cekic B, Geze S, Ozkan G, Besir A, Sonmez M, Karahan SC and Mentese A: The effect of dexmedetomidine on oxidative stress during pneumoperitoneum. Biomed Res Int 2014: 760323, 2014.

8. Ko KJ, Choi DK, Shin SJ, Ryoo HS, Kim TS, Song W, Jeon HG, Jeong BC and Seo SI: Predictive factors of prolonged warm ischemic time ( $\geq 30$ minutes) during partial nephrectomy under pneumoperitoneum. Korean J Urol 56: 742-748, 2015.

9. de Barros RF, Miranda ML, de Mattos AC, Gontijo JA, Silva VR, Iorio B and Bustorff-Silva JM: Kidney safety during surgical pneumoperitoneum: An experimental study in rats. Surg Endosc 26: 3195-3200, 2012.

10. Demyttenaere S, Feldman LS and Fried GM: Effect of pneumoperitoneum on renal perfusion and function: A systematic review. Surg Endosc 21: 152-160, 2007

11. Ozmen MM, Zulfikaroglu B, Besler TH, Col C, Cinel L and Cinel I: The correlation between reactive oxygen species and histopathology of the liver, gut, and kidneys in animals with elevated intra-abdominal pressure. J Laparoendosc Adv Surg Tech A 19: 339-343, 2009.

12. Sammour T, Mittal A, Loveday BP, Kahokehr A, Phillips AR, Windsor JA and Hill AG: Systematic review of oxidative stress associated with pneumoperitoneum. Br J Surg 96: 836-850, 2009.

13. de Seigneux S, Klopfenstein CE, Iselin C and Martin PY: The risk of acute kidney injury following laparoscopic surgery in a chronic kidney disease patient. NDT Plus 4: 339-341, 2011.

14. Dolkart O, Khoury W, Amar E and Weinbroum AA: Pneumoperitoneum in the presence of acute and chronic kidney injury: An experimental model in rats. J Urol 192: 1266-1271, 2014 .

15. National Research Council: Guide for the Care and Use of Laboratory Animals. National Acadamies Press, Washington, DC, 1996

16. Wen JG, Chen Y, Frøkiaer J, Jørgensen TM and Djurhuus JC: Experimental partial unilateral ureter obstruction. I. Pressure flow relationship in a rat model with mild and severe acute ureter obstruction. J Urol 160: 1567-1571, 1998.

17. Oyanagui Y: Reevaluation of assay methods and establishment of kit for superoxide dismutase activity. Anal Biochem 142: 290-296, 1984

18. Perelman A, Wachtel C, Cohen M, Haupt S, Shapiro H and Tzur A: JC-1: Alternative excitation wavelengths facilitate mitochondrial membrane potential cytometry. Cell Death Dis 3: e430, 2012. 
19. Makarov DV, Kainth D, Link RE and Kavoussi LR: Physiologic changes during helium insufflation in high-risk patients during laparoscopic renal procedures. Urology 70: 35-37, 2007.

20. Özdemir-van Brunschot DM, van Laarhoven KC, Scheffer GJ, Pouwels S, Wever KE and Warlé MC: What is the evidence for the use of low-pressure pneumoperitoneum? A systematic review. Surg Endosc 30: 2049-2065, 2016.

21. Wiesenthal JD, Fazio LM, Perks AE, Blew BD, Mazer D, Hare G Honey RJ and Pace KT: Effect of pneumoperitoneum on renal tissue oxygenation and blood flow in a rat model. Urology 77: 1508.e9-e15, 2011.

22. Borba M, Lopes R, Carmona M, Neto BM, Nahas SC and Pereira PR: Effects of enalaprilat on the renin-angiotensinaldosterone system and on renal function during $\mathrm{CO}_{2}$ pneumoperitoneum. J Endourol 19: 1026-1031, 2005.

23. Cay A, Imamoğlu M, Unsal MA, Aydin S, Alver A, Akyol A and Sarihan H: Does anti-oxidant prophylaxis with melatonin prevent adverse outcomes related to increased oxidative stress caused by laparoscopy in experimental rat model? J Surg Res 135: 2-8, 2006.

24. Tosun M, Yucel M, Kucuk A and Sezen S: P53 related apoptosis in kidneys in $\mathrm{CO}_{2}$ pneumoperitoneum rat model: An immunohistochemical study. Mol Biol Rep 41: 6391-6395, 2014.

25. Zhong N, Zhang Y, Zhu HF and Zhou ZN: Intermittent hypoxia exposure prevents mtDNA deletion and mitochondrial structure damage produced by ischemia/reperfusion injury. Sheng Li Xue Bao 52: 375-380, 2000.

26. Li W, Cao Z, Xia Z, Meng Q, Yu WM, Yao X and Cheng F: Acute kidney injury induced by various pneumoperitoneum pressures in a rabbit model of mild and severe hydronephrosis. Urol Int 94: 225-233, 2015

27. Cao Z, Yu W, Li W, Cheng F, Rao T, Yao X, Zhang X and Larré S Oxidative damage and mitochondrial injuries are induced by various irrigation pressures in rabbit models of mild and severe hydronephrosis. PLoS One 10: e0127143, 2015.

28. Cao Z, Yu W, Li W, Cheng F, Xia Y, Rao T, Yao X, Zhang X and Larré S: Acute kidney injuries induced by various irrigation pressures in rat models of mild and severe hydronephrosis. Urology 82: 1453.e9-16, 2013.

29. Shigeta K, Kikuchi E, Hagiwara M, Hattori S, Kaneko G Hasegawa M, Takeda T, Jinzaki M, Akita H, Miyajima A, et al: Visceral to total obesity ratio and severe hydronephrosis are independently associated with prolonged pneumoperitoneum operative time in patients undergoing laparoscopic radical nephroureterectomy for upper tract urothelial carcinoma. Springerplus 4: 290, 2015.

30. Kwak GH, Kim TH and Kim HY: Down-regulation of MsrB3 induces cancer cell apoptosis through reactive oxygen species production and intrinsic mitochondrial pathway activation. Biochem Biophys Res Commun 483: 468-474, 2017.
31. Mehaney DA, Darwish HA, Hegazy RA, Nooh MM, Tawdy AM, Gawdat HI and El-Sawalhi MM: Analysis of oxidative stress status, catalase and catechol-O-methyltransferase polymorphisms in Egyptian vitiligo patients. PLoS One 9: e99286, 2014.

32. Tortora M, Corsini S and Nistri A: Nicotinic receptors modulate the onset of reactive oxygen species production and mitochondrial dysfunction evoked by glutamate uptake block in the rat hypoglossal nucleus. Neurosci Lett 639: 43-48, 2017.

33. Wang Y, Gao H, Na XL, Dong SY, Dong HW, Yu J, Jia L and Wu YH: Aniline induces oxidative stress and apoptosis of primary cultured hepatocytes. Int J Environ Res Public Health 13: E1188, 2016.

34. Cigremis Y, Turel H, Adiguzel K, Akgoz M, Kart A, Karaman M and Ozen $\mathrm{H}$ : The effects of acute acetaminophen toxicity on hepatic mRNA expression of SOD, CAT, GSH-Px, and levels of peroxynitrite, nitric oxide, reduced glutathione, and malondialdehyde in rabbit. Mol Cell Biochem 323: 31-38, 2009.

35. Mookerjee SA and Brand MD: Measurement and analysis of extracellular acid production to determine glycolytic rate. $\mathrm{J}$ Vis Exp: e53464, 2015.

36. Sung DK, Chang YS, Kang S, Song HY, Park WS and Lee BH: Comparative evaluation of hypoxic-ischemic brain injury by flow cytometric analysis of mitochondrial membrane potential with JC-1 in neonatal rats. J Neurosci Methods 193: 232-238, 2010.

37. Wang LR, Xue X, Hu XM, Wei MY, Zhang CQ, Ge GL and Liang XJ: Structure-dependent mitochondrial dysfunction and hypoxia induced with single-walled carbon nanotubes. Small 10: 2859-2869, 2014.

38. Kroemer G, Galluzzi L and Brenner C: Mitochondrial membrane permeabilization in cell death. Physiol Rev 87: 99-163, 2007.

39. Avital S, Itah R, Szomstein S, Rosenthal R, Inbar R, Sckornik Y and Weinbroum A: Correlation of $\mathrm{CO} 2$ pneumoperitoneal pressures between rodents and humans. Surg Endosc 23: 50-54, 2009.

40. Balayssac D, Pereira B, Bazin JE, Le Roy B, Pezet D and Gagnière J: Warmed and humidified carbon dioxide for abdominal laparoscopic surgery: Meta-analysis of the current literature. Surg Endosc 31: 1-12, 2017.

41. Sammour T, Mittal A, Delahunt B, Phillips AR and Hill AG: Warming and humidification have no effect on oxidative stress during pneumoperitoneum in rats. Minim Invasive Ther Allied Technol 20: 329-337, 2011.

42. Akbulut G, Polat C, Aktepe F, Yilmaz S, Kahraman A, Serteser M, Gökçe $\mathrm{C}$ and Gökçe O: The oxidative effect of prolonged $\mathrm{CO}_{2}$ pneumoperitoneum on renal tissue of rats. Surg Endosc 18: 1384-1388, 2004. 